\title{
вмJ Global Health Impact of a personalised, digital, HIV self-testing app-based program on linkages and new infections in the township populations of South Africa
}

\author{
Nitika Pai (1) ,1,2 Aliasgar Esmail, ${ }^{3,4}$ Paramita Saha Chaudhuri, ${ }^{5}$ Suzette Oelofse, ${ }^{4}$ \\ Marietjie Pretorius, ${ }^{4}$ Gayatri Marathe, ${ }^{5}$ Jana Daher, ${ }^{2}$ Megan Smallwood, ${ }^{2,5}$ \\ Nicolaos Karatzas, ${ }^{6}$ Mohammed Fadul, ${ }^{4}$ Anna de Waal, ${ }^{2,5}$ Nora Engel, ${ }^{7}$ \\ Alice Anne Zwerling, ${ }^{8}$ Keertan Dheda ${ }^{3,4,9}$
}

To cite: Pai N, Esmail A, Saha Chaudhuri P, et al. Impact of a personalised, digital, HIV self-testing app-based program on linkages and new infections in the township populations of South Africa. BMJ Global Health 2021;6:e006032. doi:10.1136/ bmjgh-2021-006032

Handling editor Seye Abimbola

Received 16 April 2021 Accepted 15 August 2021
Check for updates

(C) Author(s) (or their employer(s)) 2021. Re-use permitted under CC BY. Published by BMJ.

For numbered affiliations see end of article.

Correspondence to Dr Nitika Pai;

Nitika.Pai@mcgill.ca

\section{ABSTRACT}

Introduction Implementation data for digital unsupervised HIV self-testing (HIVST) are sparse. We evaluated the impact of an app-based, personalised, oral HIVST program offered by healthcare workers in Western Cape, South Africa.

Methods In a quasirandomised study ( $\mathrm{n}=3095)$, we recruited consenting adults with undiagnosed HIV infection from township clinics. To the HIVST arm participants $(n=1535)$, we offered a choice of an offsite (home, office or kiosk based), unsupervised digital HIVST program ( $\mathrm{n}=962)$, or an onsite, clinic-based, supervised digital HIVST program $(n=573)$ with 24/7 linkages services.

With propensity score analyses, we compared outcomes (ie, linkages, new HIV infections and test referrals) with conventional HIV testing (ConvHT) arm participants $(n=1560)$, recruited randomly from geographically separated clinics. Results In both arms, participants were young (HIVST vs ConvHT) (mean age: 28.2 years vs 29.2 years), female $(65.0 \%$ vs $76.0 \%)$ and had monthly income $<3000$ rand $(80.8 \%$ vs $75 \%)$.

Participants chose unsupervised HIVST (62.7\%) versus supervised HIVST and reported multiple sex partners $(10.88 \%$ vs $8.7 \%)$, exposure to sex workers (1.4\% vs $0.2 \%$ ) and fewer comorbidities ( $0.9 \%$ vs $1.9 \%)$. Almost all HIVST participants were linked (unsupervised HIVST (99.7\%), supervised HIVST (99.8\%) vs ConvHT (98.5\%)) (adj RR 1.012; 95\% Cl 1.005 to 1.018) with new HIV infections: overall HIVST (9\%); supervised HIVST (10.9\%) and unsupervised HIVST (7.6\%) versus ConvHT (6.79\%) (adj RR 1.305; 95\% Cl 1.023 to 1.665); test referrals: $16.7 \%$ HIVST versus $3.1 \%$ ConvHT (adj RR $5.435 ; 95 \% \mathrm{Cl}$ 4.024 to 7.340 ).

Conclusions Our flexible, personalised, app-based HIVST program, offered by healthcare workers, successfully linked almost all HIV self-testers, detected new infections and increased referrals to self-test. Data are relevant for digital HIVST initiatives worldwide.

\section{INTRODUCTION}

\section{Background and rationale}

In 2016, the WHO recommended HIV selftesting (HIVST) for individuals living with

\section{Key questions}

What is already known?

- The WHO has called for evidence on data for digital supports and use of community-based healthcare workers to improve services associated with HIV self-testing (HIVST).

- Data for HIVST with digital supports from Southern Africa, especially with app-based programs, remain sparse.

What are the new findings?

- Our healthcare workers offered a flexible, personalised program, with choice of venue and strategy, customised to preferences of participants.

- Participants who showed up to test in clinics were recruited. We also documented the standard of care in the neighbouring clinics.

- Over an 18-month period, we linked all positive and negative unsupervised HIVST and supervised HIVST participants to antiretroviral treatment initiation and preventative care pathways. We detected new infections and referrals to self-test with the program.

\section{What do the new findings imply?}

- This quasirandomised transition-to-scale real-life study that mimicked a real-life implementation of HIVST with digital supports.

- Findings imply a possibility to link participants to care, with a trained corpus of healthcare workers, and a 24/7 linkage service to counselling, prevention and treatment initiation through a personalised, anonymized, secure, app-based digital program.

- Findings are relevant for global stakeholders who wish to deploy such programs to young, digitally savvy populations in the region. It offers data to guide scale up of such strategies for HIV, related coinfections and COVID-19.

undiagnosed HIV infection to know their serostatus. ${ }^{1}$ Performed with an approved selftest, HIVST offered preliminary screening test results, and laboratory-based confirmatory testing was deemed essential. ${ }^{1-4}$ Research 
evidence on HIVST has accumulated exponentially ${ }^{5-7}$ with reported increases in self-test uptake, ${ }^{8-10}$ detection of new HIV infections, increases in test frequency and partner referrals. ${ }^{11-13}$

Recently, the WHO has called for evidence in HIVST in the following areas: (A) digital innovations, (B) community health workers and peer counsellors for operationalising linkages and $(\mathrm{C})$ social network use for key populations. ${ }^{14}$ Global data on scalable HIVST service delivery models with digital support tools that work in real-life settings remain sparse. $^{15-17}$

With digital support tools, linkages to antiretroviral treatment (ART) and prevention services (ie, pre-exposure prophylaxis, partner notification and medical circumcision), tracking and surveillance are possible. ${ }^{18-20}$ With COVID-19 induced lockdowns and restrictions faced globally, the demand for digital tools has grown exponentially in countries. ${ }^{18-20}$ With an increasing availability of $4 \mathrm{G} / 5 \mathrm{G}$ networks, together with an increase in ownership of smartphones/tablets in low and middle-income countries, there is an opportunity to scale digital solutions.

Global foundations and initiatives are looking for evidence to scale HIVST strategies/programs with digital support tools; yet for South Africa, data and evidence remain sparse. Furthermore, the impact of preferred choice of HIVST strategy, venue, and customized linkages, offered by health care workers and peer counsellors has never been explored.

To address these evidence gaps, we report an evaluation of a choice-based, personalised digital app/platform-based HIVST program, offered by healthcare workers and peer counsellors, in the township communities of Cape Town, South Africa.

\section{Objectives}

We set out to compare an offer of our oral HIVST program with digital supports, offered as supervised or unsupervised HIVST, to conventional HIV testing (ConvHT) (conventional rapid and lab tests+onsite counsellor) on impact outcomes (ie, linkages, new infections and referrals to test).

\section{Hypotheses}

We conservatively hypothesised that the proportion of participants (self-testers) linked to post-test counselling and care (ie, ART initiation) and related prevention services (ie, medical circumcision, pre-exposure prophylaxis and partner notification) and the proportion of new infections detected in self testers will be comparable in both ConvHT and HIVST arms, whereas the proportion of self-test referrals (within/to social networks) in the HIVST arm would be higher (twofold, due to its ability to reach testers) than the ConvHT arm.

\section{METHODS}

\section{Eligibility criteria}

Included participants were 18 years or older, of unknown HIV status at baseline (past 3 months), with access to an Android/iPhone smartphone or ability to use a tablet/ smartphone for HIVST. Excluded participants were either on antiretroviral therapy (ART), or with a confirmed HIV diagnosis, or a serious medical condition requiring hospitalisation.

\section{Study design, recruitment setting and location}

The evaluation was conducted in Cape Town, South Africa, between January 2017 and June 2018. A community clinic-based quasirandomised trial study design was used to generate real-life field implementation data with an intention to scale the intervention on completion.

Convenience sampling was used to recruit participants. Members of the township population presenting to test for HIV by self-referral or referral by self-testers were recruited.

\section{Patient and public involvement}

Participants or the public were not involved in the design, or conduct, or reporting, or dissemination plans of our research.

\section{Recruitment/sampling}

We created a sampling frame of all clinics served by the University of Cape Town. Before study initiation, we geomapped all the districts in Western Cape and generated a random number sequence in STATA V.12. Within each district, from the sampling frame, we randomly sampled two geographically separated clinics to offer either HIVST or ConvHT for a total of six clinics in all.

Our study recruitment period was from January 2017 to June 2018.

We recruited individuals who presented for HIV testing to the community outreach clinics and those who met the eligibility criteria. Study participants were encouraged to refer self-testing to their partners, friends and family within their close social networks. Individuals referred by self-testers that participated in the study were also recruited. Our clinic staff recruited participants during routine and drop-in visits.

For study promotions, community outreach by healthcare workers, word of mouth, handouts/flyers, demonstration videos in clinics, a Facebook page and radio/TV announcements were deployed.

\section{Designing and developing the innovation}

In 2009, in Canada, we first developed a web-based HIVST strategy using design thinking principles and evaluated a prototype for usability (HIVSmart!, McGill University, 2013).$^{21}$ Subsequently, we evaluated the strategy for feasibility in South Africa ${ }^{22}$ and Canada. ${ }^{2324}$ Funded by the Governments of Canada and South Africa, we expanded our strategy to a digital HIVST app-based program of care in 2015 (figure 1). We added the following features for personalisation: multilingual content in five languages (ie, Xhosa, Afrikaans, Zulu, English and French); a device agnostic, Android/iOS portable platform (ie, tablet, web, smart/mobile phone); added simplified video instructions for HIVST; self-test interpretation/capture with data storage; and a 24/7 preferred clinic based service 


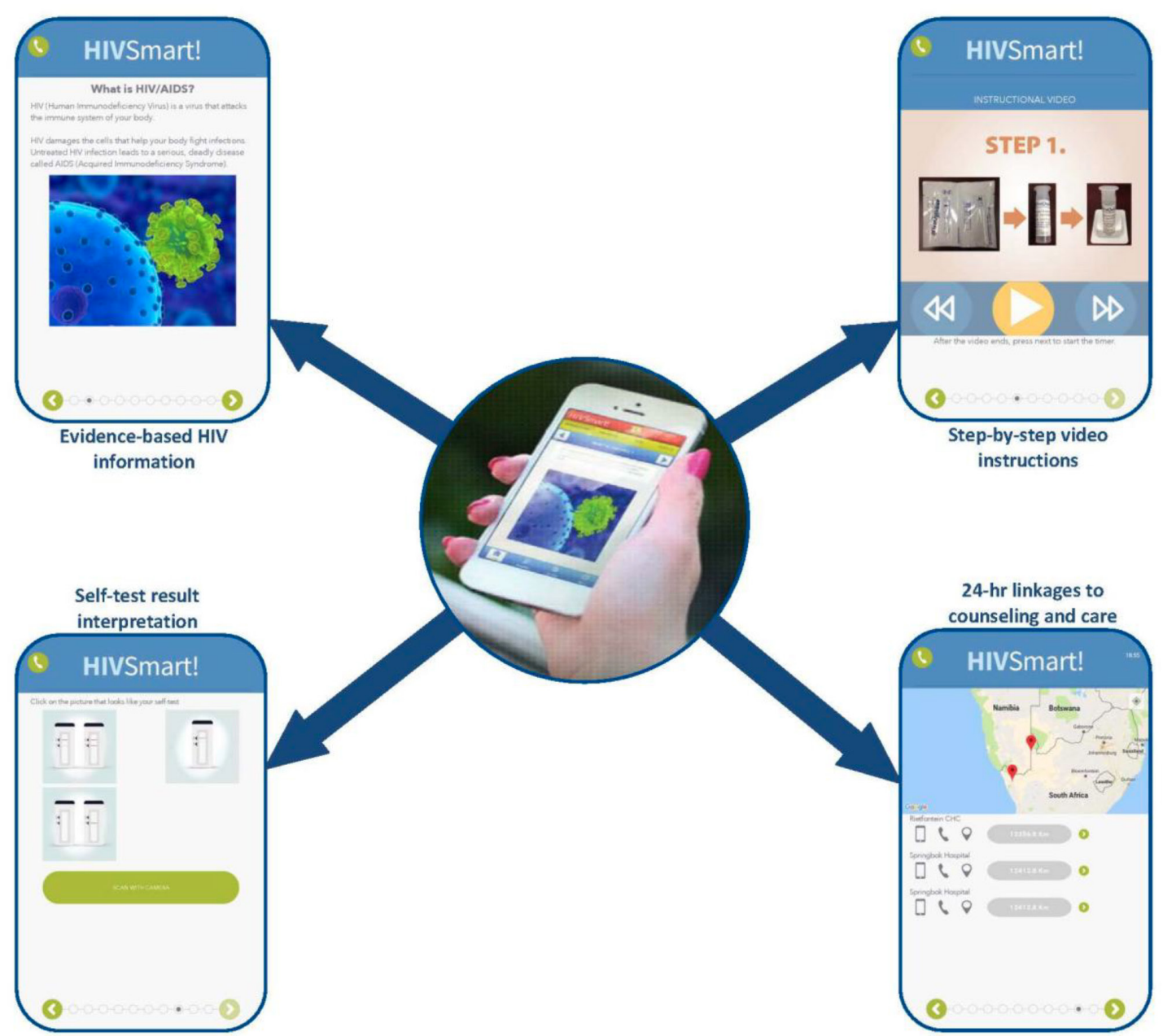

Figure 1 The HIVSmart! app-based digital program of care operated by healthcare workers and peer counsellors in Cape Town, South Africa.

that offered pretest/post-test counselling/linkage service to treatment and prevention pathways, in their preferred language, to nearby clinics. The counselling and linkage service was offered $24 / 7$ by trained healthcare workers and peer counsellors (ie, over phone calls, texts, chats, messages and face to face). We housed our program on a secure Health Insurance Portability and Accountability Act (HIPAA)-compliant cloud server and added a user-friendly dashboard and a global positioning system clinic locator, which was useful to both patients and providers. $^{22} 23$

\section{HIVST arm}

Our intervention consisted of our innovative digital HIVST program together with an oral HIV self-test (OraQuick advance HIV-1/2, OraSure Technologies Inc, USA). Our mobile app was downloaded onto the participant's phone or tablet (using QR codes). Participants were provided a Wi-Fi access card for connectivity.

From each participant, the research nurse obtained informed consent, explained the study procedure, performed standard per clinic protocols (rapid tests and reference standard testing for the labs) to provide consistent reference standard data and collected data on sociodemographic and test experience.

\section{Delivery of the intervention}

We offered a choice of two strategies and associated venues to self-test, together with customised linkages to preferred clinics for ART initiation and follow-up chosen by participants. No financial incentives and no phones were offered to study participants. Only Wi-Fi access cards for connectivity were provided to make it easy for the participants to communicate with the healthcare workers through the app via text messages/calls. The phones belonged to the participants. Research staff who did not own phones were provided with smartphones and a Wi-Fi access card for the entire duration of the study for communication purposes only.

The procedures for each HIVST option to test were as follows:

1. Supervised digital HIVST at the clinic: participants performed HIVST in the presence of a counsellor or healthcare worker. For this option, participants were provided a tablet with the downloaded app, an oral self-test and a Wi-Fi access card to call counsellors. 
Participants were ushered to a private space set up in the clinic to perform the self-test. After self-test was conducted, participants could drop their self-test in a drop box outside the clinic. Total duration of intervention was 1.5 hours (including orientation and testing). The tablet was housed in the clinic and was secured with a lock to prevent it from being taken home by anyone. Tablets were collected from the clinic at the end of the day and stored in a locked drawer in the offices of the principal investigator.

2. Unsupervised digital HIVST at a venue of their choice: the research nurse offered participants the option to conduct their self-tests unsupervised anywhere: at home, at their office or workspace or in private space (kiosk). The participants were provided with an oral self-test, a QR code and a Wi-Fi access card to call counsellors. Orientation time of $30 \mathrm{~min}$, and unsupervised test program of 1 hour, for a total duration of 1.5 hours.

The phones belonged to the participants and were not provided to the participants. Participants were asked to download the QR code in the presence of the counsellor to check if the app was working. Once the app opened up, the participants were allowed to leave. Participants who were confident of testing by themselves chose the home/office option, and participants who lacked safe space to test at home or office chose to test at the kiosk. Participants were requested to drop the test kits back at the clinic in the drop off box, or bring it with them when they came back to the counsellor for care.

To arrange for linkages to counselling, disease staging or ART initiation in test positives and prevention services in test negatives, the healthcare workers/peer counsellors recorded participant's language preferences, preferred mode of follow-up communication (chat, SMS, phone call and face to face) and their preferred clinic location (UCT vs non-UCT). This helped the healthcare workers customise their service to the choice, preference and lifestyle of participants.

\section{ConvHT arm}

ConvHT testing (standard of care standard rapid and lab tests) was offered in comparator clinics with onsite counsellors; duration was 1.5-2 hours with 3-4hours of waiting time in clinics. The research nurses explained the study procedure to consenting participants, obtained informed consent and performed ConvHT (blood-based rapid tests and sample collection for reference standard), followed by data collection for sociodemographic variables and test experience. Participants were asked to refer their partners and friends and family to test for HIV at these clinics. Linkage and counselling service followed the model used for the ConvHT conducted in two steps. The first step in linkage seeking is post-test counselling and sharing of lab confirmed test result. This is followed by blood draw for disease staging of lab confirmed test positives and risk reduction counselling and triage to prevention services (ie, medical circumcision, preexposure prophylaxis and partner notification) for lab confirmed test negatives. The second step was ART initiation at the clinic based on all lab results for test positives.

\section{Outcomes}

To evaluate the impact on the primary outcome, linkages to counselling, ART initiation and prevention services, we documented comparative linkage data from the ConvHT arm.

Linkage was defined in two ways:

1. Linkage to counselling, a proportion, was computed by documenting numerator/denominator. Numerator was compiuted as the number of both self-test positive and self-test negative participants who communicated and showed up for face-to-face post-test counselling, receipt of lab-confirmed test results, followed by disease staging of lab confirmed test positives, or triage to prevention services for lab confirmed test negatives. Denominator was defined as the total number of consenting participants who sought self testing.

2. Linkages to care was defined as the proportion of newly identified participants linked to clinics for ART initiation (numerator) over total number of test positives identified in the study (denominator).

Proportion of linkages in each arm were documented, and comparisons were reported with risk ratio (RR) and associated $95 \%$ CIs.

For the HIVST arm, we recorded receipt of linkage services through the application; when a participant called for counselling, a phone call or virtual chat occurred, followed by a face-to-face meeting for services. Linkages to treatment for positives in preferred clinics were confirmed by date and corroborated with record of receipt of ART from clinic rosters by healthcare workers. Similarly, linkages to prevention services for test negatives were confirmed and corroborated with clinic records maintained by healthcare workers.

To evaluate the impact on the secondary outcome, detection of new infections, we compared and documented the proportion of newly identified participants as test positives in both arms. Test positives/negatives were confirmed by lab-confirmed results and test protocols. For both arms, we calculated the total number of new infections detected and staged (numerator) over the total number of individuals that sought testing (denominator). To compare new infections, we considered the test results (against a reference standard of rapid tests and lab-confirmed tests) separately, and computed RR $(95 \% \mathrm{CI})$.

To evaluate the impact on tertiary outcome, referrals to HIVST, separately for each arm (HIVST and ConvHT), we computed the total number of referrals to test made by a self-tester (phone-based and word-of-mouth referrals) to someone in their social network (ie, partners, spouses, friends, family and community members). Next, for each arm, we compared the differences in estimates from the end of the study to baseline. Referrals were 
documented by our research nurses who explained the choices of strategy or venues to test and inquired about their reasons and motivations for referrals. Referrals were not linked to primary self-tester to avoid a breach of confidentiality. Proportions were compared, and RRs (95\% CI) were computed.

To document choice, we computed the proportions by calculating the total number of consenting HIVST participants (denominator). We calculated HIVST participants who chose either strategy, supervised HIVST(one venue: clinic based), versus, unsupervised HIVST (three venues: home, office/prviate space and kiosk) for the numerator.

\section{Sample size}

Assuming an equivalence margin of $10 \%$, success proportion for linkage of $85 \%$ for HIVST arm and $90 \%$ for the conventional testing arm (alpha 5\%; power of 95\%), a sample size of 2262 participants (1131 in each arm) was deemed sufficient for our linkage estimations. Assuming an attrition rate of $10 \%$, sample size of 1500 per cohort was estimated to be adequate to detect comparable linkages and new infections between HIVST and ConvHT and to detect a conservative twofold increase in referral in the HIVST cohort compared with ConvHT cohort, with a $\mathrm{CI} \pm 10 \%$.

\section{Assignment method}

The unit of assignment was the individual participant. Although our participants, investigators and staff were open (unblinded) to the intervention, outcome assessors (statisticians) were blinded to the intervention assignment. Assignments were revealed to them at the end of their analyses. Assignment was restricted to clinics served by University of Cape Town.

\section{Data sources, collection and measurement}

For deidentification of each participant, a unique study ID number was created. It identified the site, the township clinic and the program (HIVST or ConvHT). Likewise, each self-test referral was coded by a reference number that identified the site, the township clinic and the program, self-reported by the study participant and recorded.

For both arms, we collected deidentified, encrypted, digital data on the app linked reference standard laboratory test data. For the ConvHT arm, baseline characteristics (table 1) data were collected using digital case report forms with anonymised data on exposure, outcome and confounders. Laboratory data collected on tablets were supplemented by data from HIV registers/folders.

For the HIVST arm, anonymised data on exposure, outcome, confounders, laboratory data, review of HIV registers, encrypted data on the app and the server were similarly collected. Access to the HIPAA-compliant platform was granted only to the two principal investigators involved in the study. Each day, data from the app were corroborated with lab data and clinic data. Access to recruiting staff was restricted to data entry only.
A regular oversight and monitoring of recruitment was possible with a dashboard. Daily record of numbers improved data quality and provided a snapshot to the key personnel involved in the study. Data on ART clinic initiation and lab results were corroborated with clinic rosters. Some participants preferred to link to clinics outside the University of Cape Town (UCT) system. To allow for this, permission was sought from the city to collect data from non-UCT clinics by study staff. All self-tests were confirmed by rapid tests and laboratory tests as per clinic protocols.

\section{Statistical methods}

A descriptive analysis on characteristics of all study participants was performed. For the continuous covariates, we reported mean $(\mathrm{SD})$, and for categorical covariates, we reported \% in each category. We replaced missing covariate values by the most frequent category (categorical variables) or the median (continuous variables).

We considered outcome metrics (ie, linkages, new infections and referrals) as binary and defined success and failure appropriately. For each outcome, we compared the proportion of success between the HIVST and ConvHT arms. We performed the statistical z-test to compare two proportions, and we reported the RR and 95\% CI for our outcomes.

An RR $>1$ indicated that the HIVST arms were favourable (linkage, new infections and higher referral), while an $\mathrm{RR}<1$ indicated that the ConvHT arm was favourable.

To control for confounding at baseline (to reduce selection bias), we performed a propensity-matched analysis of outcomes. Variables for the propensity model (ie, age, gender, Socio-economic status (SES), work status, sexual history and comorbidities) were decided a priori based on their significance and their impact on participant's choice and preference. A nearest-neighbour matching criterion was used to identify comparable subjects in each cohort. Following the identification of the matched cohort, we estimated the RR and CI for each of the outcomes as mentioned by Austin ${ }^{25}$ and performed our Statistical analyses in R (V.3.3.0).

\section{RESULTS \\ Demographics and flow}

A total of 3137 participants were enrolled, of which 33 in the self-testing arm and nine in the conventional arm opted out (reasons are documented in figure 2).

Of the 3095 participants, 1535 participants in the HIVST arm and 1560 participants in the ConvHT were recruited, respectively. A majority of participants in the HIVST arm ( $\mathrm{n}=962,62.7 \%)$ chose unsupervised HIVST over supervised HIVST $(\mathrm{n}=573,37.3 \%)$.

Sociodemographic data on participants in HIVST versus ConvHT arm demonstrates that participants in both arms were young (mean age: 28.2 years vs 29.2 years), female $(65.0 \%$ vs $76.0 \%)$ and poor $<3000$ rand (US\$253) $(80.8 \%$ vs $75.0 \%)$. 
BMJ Global Health

Table 1 Baseline characteristics of participants

\begin{tabular}{|c|c|c|c|}
\hline Characteristics & $\begin{array}{l}\text { Self-testing arm } \\
(n=1535)\end{array}$ & $\begin{array}{l}\text { Conventional testing arm } \\
(n=1560)\end{array}$ & $\begin{array}{l}\text { Difference } \\
(95 \% \mathrm{Cl})\end{array}$ \\
\hline Age, mean (SD) & $28.23(8.83)$ & $29.18(8.56)$ & $-0.95(-1.56$ to 0.34$)$ \\
\hline $\mathrm{NA}=\operatorname{missing}(\mathrm{n}(\%))$ & $0(0.00)$ & $1(0.06)$ & $-0.0006(-0.0018$ to 0.0006$)$ \\
\hline $\begin{array}{l}\text { Gender } \\
\text { (n (\%) male) }\end{array}$ & $541(35.24)$ & $378(24.23)$ & $0.11(0.08 \text { to } 0.14)^{*}$ \\
\hline$N A=$ missing & $0(0.00)$ & $1(0.06)$ & $-0.0006(-0.0018$ to 0.0006$)$ \\
\hline \multicolumn{4}{|l|}{ Education (n (\%)) } \\
\hline $0=$ no schooling OR primary school & $114(7.43)$ & $100(6.41)$ & $0.01(-0.01$ to 0.03$)$ \\
\hline 1=high school OR more advanced & $1420(92.51)$ & $1460(93.59)$ & $-0.01(-0.03$ to 0.01$)$ \\
\hline$N A=$ missing & $1(0.07 \%)$ & $0(0.00 \%)$ & $0.0007(-0.0006$ to 0.0020$)$ \\
\hline \multicolumn{4}{|l|}{ Work situations (n (\%)) } \\
\hline $0=$ employed (full time) & $337(21.95)$ & $567(36.35)$ & $-0.14(-0.18 \text { to } 0.11)^{*}$ \\
\hline $1=$ employed (part time) & $207(13.49)$ & $177(11.35)$ & $0.02(-0.002$ to 0.044$)$ \\
\hline $2=$ not employed & $916(59.67)$ & $812(52.05)$ & $0.07(0.04 \text { to } 0.11)^{\star}$ \\
\hline $3=$ retired & $12(0.78)$ & $4(0.26)$ & $0.005(0.0001 \text { to } 0.0103)^{\star}$ \\
\hline$N A=$ missing & $63(4.10)$ & $0(0.00)$ & $0.041(0.031 \text { to } 0.051)^{*}$ \\
\hline \multicolumn{4}{|l|}{ Monthly income (n (\%)) } \\
\hline $0=<3000$ rand & $1190(77.52 \%)$ & $1167(74.81)$ & $0.03(-0.002$ to 0.06$)$ \\
\hline $1=3000-6000$ rand & $153(9.97)$ & $304(19.49)$ & $-0.09(-0.12 \text { to } 0.07)^{*}$ \\
\hline $2=6000-9000$ rand & $61(3.97)$ & $46(2.95)$ & $0.01(-0.003$ to 0.023$)$ \\
\hline $3=>9000 \mathrm{rRand}$ & $68(4.43)$ & $40(2.56)$ & $0.02(0.01 \text { to } 0.03)^{*}$ \\
\hline$N A=$ missing & $63(4.10)$ & $3(0.19)$ & $0.04(0.03 \text { to } 0.05)^{*}$ \\
\hline \multicolumn{4}{|l|}{ Source of income (n (\%)) } \\
\hline $0=$ employed & $531(34.59)$ & $742(47.52)$ & $-0.13(-0.16 \text { to } 0.09)^{*}$ \\
\hline $1=$ family or friends & $947(61.69)$ & $766(49.10)$ & $0.13(0.09 \text { to } 0.16)^{*}$ \\
\hline $2=$ grant & $47(3.06)$ & $46(2.95)$ & $0.001(-0.01$ to 0.01$)$ \\
\hline $3=$ pension & $9(0.59)$ & $6(0.38)$ & $0.002(-0.003$ to 0.006$)$ \\
\hline$N A=$ missing & $1(0.07)$ & $0(0.00)$ & $0.007(-0.0006$ to 0.0020$)$ \\
\hline \multicolumn{4}{|l|}{ Previous HIV test (n (\%)) } \\
\hline $0=$ first-ever HIV test & $75(4.89)$ & $58(3.72)$ & $0.01(-0.002$ to 0.026$)$ \\
\hline $1=$ repeat HIV test (last test less than 1 year ago) & $915(59.61)$ & $1021(65.45)$ & $-0.06(-0.09 \text { to } 0.02)^{*}$ \\
\hline $2=$ repeat HIV test (last test more than 1 year ago) & $544(35.44)$ & $481(30.83)$ & $0.05(0.01 \text { to } 0.08)^{*}$ \\
\hline$N A=$ missing & $1(0.07)$ & $0(0.00)$ & $0.0007(-0.0006$ to 0.0020$)$ \\
\hline $\begin{array}{l}\text { Current partner your husband or wife? } \\
\text { (n (\%) yes) }\end{array}$ & $429(27.95)$ & $432(27.69)$ & $0.002(-0.03$ to 0.03$)$ \\
\hline$N A=$ missing & $69(4.50)$ & $12(0.77)$ & $0.04(0.03 \text { to } 0.05)^{*}$ \\
\hline Are you sexually active? (n (\%) yes) & $1223(79.67)$ & $1481(94.94)$ & $-0.15(-0.17 \text { to } 0.13)^{\star}$ \\
\hline$N A=$ missing & $74(4.82)$ & $0(0.00)$ & $0.05(0.04 \text { to } 0.06)^{*}$ \\
\hline $\begin{array}{l}\text { In the past } 6 \text { months, sex with multiple partners } \\
\text { (n (\%) yes) }\end{array}$ & $167(10.88)$ & $136(8.72$ & $0.02(0.001 \text { to } 0.042)^{*}$ \\
\hline$N A=$ missing & $81(5.28)$ & $0(0.00)$ & $0.05(0.04 \text { to } 0.06)^{*}$ \\
\hline $\begin{array}{l}\text { In the past } 6 \text { months, sex with a sex worker (n } \\
\text { (\%) yes) }\end{array}$ & $21(1.37)$ & $3(0.19)$ & $0.01(0.005 \text { to } 0.018)^{\star}$ \\
\hline$N A=$ missing $(\%)$ & $81(5.28)$ & $0(0.00)$ & 0.05 (0.04 to 0.06 ) \\
\hline $\begin{array}{l}\text { In the past } 6 \text { months, sex with an HIV-infected } \\
\text { partner (n (\%) yes) }\end{array}$ & $42(2.74)$ & $35(2.24)$ & $0.005(-0.006$ to 0.015$)$ \\
\hline$N A=$ missing & $81(5.28)$ & $0(0.00)$ & 0.05 (0.04 to 0.06$)$ \\
\hline
\end{tabular}

Have you ever been diagnosed with: 
Table 1 Continued

Characteristics

Tuberculosis (n (\%) yes) *

Other lung infection (n (\%) yes) *

Diabetes (n (\%) yes)

Hypertension (n (\%) yes) *

Asthma (n (\%) yes) *

${ }^{*}$ No missing data in both arms.

Participants in the HIVST arm versus ConvHT arm reported significant differences in the following variables: sex with multiple partners ( $11.5 \%$ vs $8.7 \%)$, exposure to sex workers $(1.4 \%$ vs $0.2 \%)$, less sexual actiivty $(79.6 \%$ vs $94.9 \%)$, with repeat HIV test more than a year $(35.4 \%$ vs $30.8 \%)$, repeat HIV test less than a year (59.6\% vs $64.5 \%)$ and HIVST participants versus ConvHT arm participants were also relatively healthier with fewer comorbidities (diabetes: $0.9 \%$ vs $1.9 \%$; hypertension: $2.9 \%$ vs $8.2 \%$ and asthma: $2.35 \%$ vs $5.19 \%$ ). More men attempted to know their status through HIVST versus ConvHT $(35.24 \%$ vs 24. $23 \%$ ).

Please refer to table 1 for baseline sociodemographic characteristics.

\section{Outcomes}

Compared with ConvHT (98.5\%), in the HIVST arm, our primary linkage outcome to counselling and care was high at $99.7 \%$ (break up: unsupervised HIVST arm: 99.8\%; supervised HIVST arm at 99.3\%) (unadjusted RR: 1.012; 95\% CI 1.005 to 1.018; propensity-adjusted RR: 1.011; $95 \%$ CI 1.005 to 1.018). Our secondary linkage outcome for ART initiation for HIV test positives and prevention pathways for HIV negatives was ((overall digital HIVST: 98.1\%; supervised HIVST: 95.7\%; unsupervised HIVST: 99.3\%) ) and comparable with ConvHT: $98.5 \%$.

At 1 month, we documented the losses to follow-up in each arm. In the HIVST arm, we had 38 participants

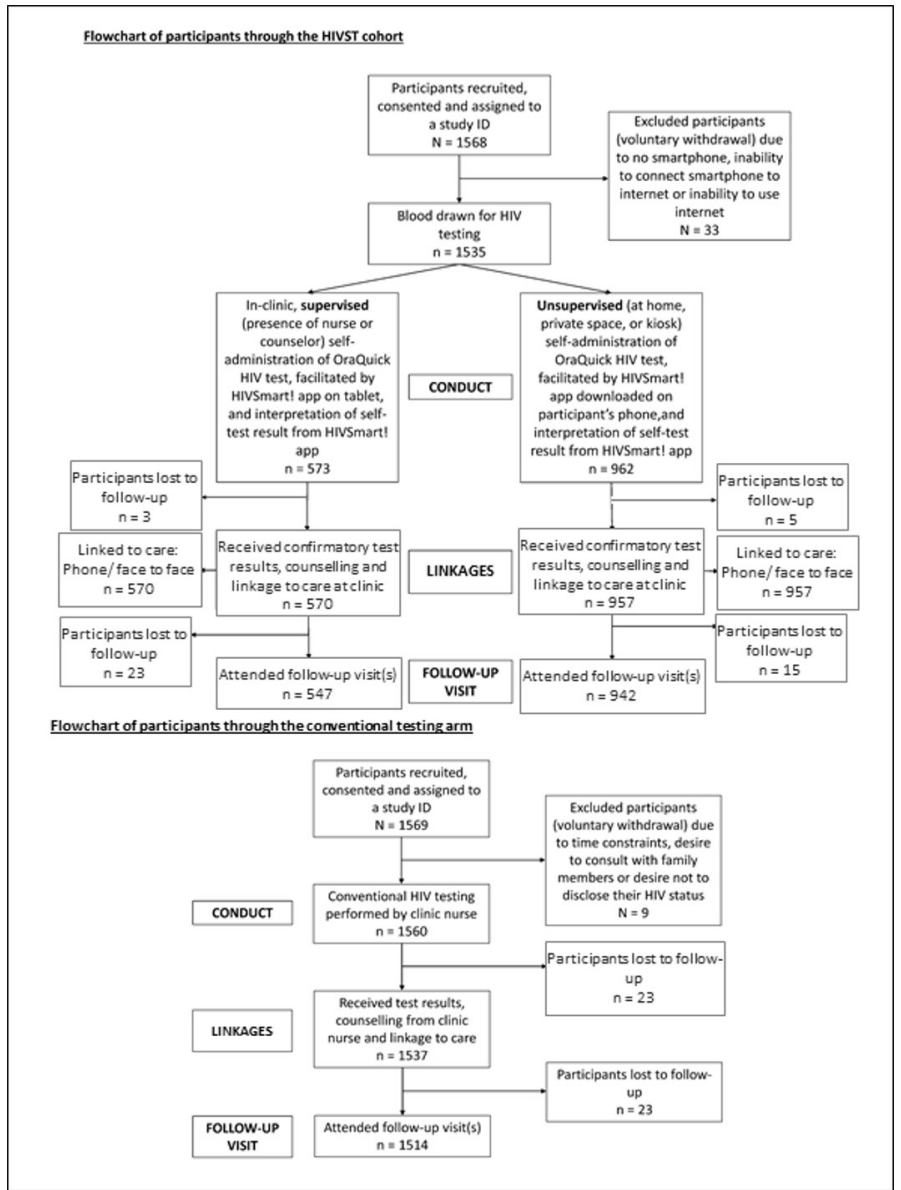

Figure 2 Flow diagram of participants through the HIVST and ConvHT arms. ConvHT, conventional HIV testing; HIVST, HIV self-testing. 
Table 2 Multivariate analyses and propensity score matched analyses.

A: multivariate analyses; adjusted risk ratio (RR) and risk differences with $95 \% \mathrm{Cl}$ for outcomes of interest; reference group conventional arm

\begin{tabular}{lll}
\hline & $\mathbf{R R}$ & $\mathbf{9 5 \%} \mathbf{C l}$ \\
\hline Linkages & 1.012 & 1.005 to 1.018 \\
New infections & 1.305 & 1.023 to 1.665 \\
Referral & 5.435 & 4.024 to 7.340 \\
\hline
\end{tabular}

B: propensity-matched analyses; adjusted RR with $95 \% \mathrm{Cl}$ for outcomes of interest

\begin{tabular}{lll}
\hline & \multicolumn{2}{l}{ Propensity adjusted } \\
\cline { 2 - 3 } & $\mathbf{R R}$ & $\mathbf{9 5 \%} \mathbf{C l}$ \\
\hline Linkage & 1.011 & 1.005 to 1.018 \\
New infections & 1.295 & 1.011 to 1.660 \\
Referral & 5.391 & 3.992 to 7.281 \\
\hline
\end{tabular}

(break up: 23 supervised, 15 unsupervised arm) versus 23 participants in the ConvHT arm.

Regarding new infections, compared with ConvHT arm at $106 / 1560(6.79 \%)$, we detected a slightly overall higher proportion (9\%) in the HIVST arm (break up: unsupervised HIVST at 73/962 (7.6\%) and supervised HIVST at $63 / 573$ (10.9\%)) (RR: $1.305 ; 95 \%$ CI 1.023 to 1.665). Propensity-adjusted analysis showed marginally better performance in the HIVST arm versus ConvHT (RR: 1.295 ; 95\% CI 1.011 to 1.665 ).

As for referrals to self-test within their social network (ie, partners, family and friends), it was proportionally higher in the HIVST arm $(16.7 \%)$ versus ConvHT arm $(3.1 \%$ ) (RR: 5.435 ; 95\% CI 4.024 to 7.340 ). The propensity-adjusted analysis preserved the RR (adjusted RR: 5.391; 95\% CI 3.992 to 7.281 ).

Regarding choice, an overwhelming majority ( $\mathrm{n}=962$ $(62.67 \%))$ chose the unsupervised HIVST strategy, while a few $(\mathrm{n}=573(37 \%))$ chose supervised HIVST. Participants were 1.70 times more likely to choose the unsupervised HIVST over supervised HIVST, reflecting the popularity of our digital unsupervised HIVST strategy. Among choice of venue to test unsupervised, homes and kiosks were popular followed by offices/workspaces.

Please refer to table 2A,B for analyses, respectively.

\section{DISCUSSION}

To the participants, we offered a personalised, choicebased HIVST program with digital support tools, in a real-life implementation study design, and compared impact outcomes in HIVST versus ConvHT arms.

First, many participants in the HIVST arm $(n=962$ participants, $62.7 \%$ ) chose unsupervised over supervised HIVST strategy, reflecting the need for autonomy, privacy and convenience to self-test. Compared with ConvHT $(98.5 \%)$, our linkage proportions for counselling for HIVST were at $(99.7 \%$ overall). Break up of linkage metrics for HIVST: unsupervised digital HIVST (99.8\%), supervised digital HIVST (99.3\%). Our linkage proportions for ART initiation in HIVST arm were comparable with ConvHT, reflecting the fact that our participants preferred the customised program offered by our healthcare workers.

Operationalising linkages has been a huge challenge in HIVST. Globally, linkage data for digital unsupervised HIVST with app-based programs are sparse. Linkage data for HIVST (without a digital component) varies between $51 \%$ and $81 \%^{1}$ and has been reported from many countries: Thailand ${ }^{26}$ China,${ }^{27}{ }^{28}$ Vietnam,${ }^{29}$ Singapore,${ }^{30}$ the Americas (Brazil, ${ }^{31}$ USA $^{32-34}$ and Canada), ${ }^{2324}$ Australia $^{35}$ and Europe (Spain). ${ }^{36}$ We believe that our customised, unincentivised, 24/7 digital program offered by our healthcare workers, together with a choice of strategy and venue to test, contributed to the success of our strategy. ${ }^{37}$ HIVST strategy was popular, but unsupervised HIVST appealed to the participants that wanted to exercise privacy, autonomy and independence to test anywhere. Although homes, offices were popular venues to self-test, those participants who did not find homes/offices to be safe/comfortable preferred kiosks. Exploration of choice of strategy and venue in the context of this study has been examined in great detail in related recently published qualitative research from this project. ${ }^{38} 39$

Retention in care was also maintained throughout the study period through digital communication. This was not entirely surprising, for we found similar retention in care estimates with our AideSmart! Multiplex app-based program for coinfections for pregnant women in rural India. ${ }^{40}$ Retention in care in participants was possible without incentives because of a personalised service available $24 / 7$ that catered to their lifestyle and accommodated their choice and preferences that further enabled its success.

Second, although we detected a modest increase in new infections in the HIVST arm versus ConvHT arm (RR: $1.305 ; 95 \%$ CI 1.023 to 1.665 ), we detected many new HIV infections in both supervised and unsupervised HIVST arms; this finding concurs with cumulative evidence in HIVST. ${ }^{1}$ HIV incidence is explained by the popularity of the strategy in young populations. The HIV incidence was high in young women and their referrals. Perhaps, there is a need for targeted, digital strategies to engage these young digitally savvy populations. ${ }^{41}$

Third, we extended referrals to partners, friends and family within their social network, including those in need of HIV testing and those living with undiagnosed HIV infection and those that were not keen to test in conventional clinics. ${ }^{42}{ }^{43}$ With that, we found a high proportion of referrals for HIVST. This finding is in line with WHO recommendations for HIVST. Secondary distribution of self-tests to partners has been successful in HIVST. ${ }^{44}$ Incidentally, our self-testers were predominantly young women, but our referrals were predominantly men. Rapid information sharing by women to men was possible through our digital HIVST app that actively 
engaged young men. ${ }^{45}$ We concur that women could be the change agents for HIVST- by encouraging men within their social network to self-test, with a digital program. they can expedite progress towards UNAIDS targets. Our finding is aligned with HIVST studies ${ }^{4346}$ and supports current WHO recommendations.

A few elements of our HIVST program have contributed to its success. These are: (A) deployment of a complete app-based program with digital supports, (B) offering a choice of strategy, a choice of venue to selftest and a choice to link participants to their preferred clinics for ART and prevention pathways, (C) provision of customised communication in the participant's language, together with a choice of communication modalities (ie, text, calls, chat, followed by face to face), encouraged a bidirectional exchange impacting participant engagement; (D) continued presence of our team of healthcare workers with a $24 / 7$ service that included facilitating counselling, linkage to ART initiation at the nearest preferred clinic, facilitating follow-up and linkages to prevention services; and lastly (E) task shifting of counsellors and healthcare workers, from performing primary screening in clinics to optimising linkages. ${ }^{46}$

\section{Strengths}

Our study strengths lay in its novelty, its digital program, its large sample size, representative digitally savvy young populations and flexible real-life implementation study design. A quasirandomised study design offered the flexibility to understand choice of strategy, venue to test and customisation of counselling and linkage services based on self-tester's preference and comfort level with technology. It was not possible to explore choice nor offer flexibility, within a randomised clinical trial framework. Furthermore, many such flexible designs are now being recommended for digital initiatives. ${ }^{48}$ We have conducted extensive qualitative research to explore the role of choice, flexibility and preferences, and customisation in detail. ${ }^{38}{ }^{39}$ We are also completing a cost-effectiveness analysis. ${ }^{49}$

\section{Limitations}

Our data are comparable, as confirmed by negligible baseline differences (balance) in the participants' demographics minimising sampling bias. A random selection of study and comparator sites in the design stage minimised selection bias. A complete reporting of all deidentified outcomes and blinding our statisticians to the intervention assignment minimised detection bias. Propensityadjusted modelling minimised confounding at baseline. Propensity scores do not account for latent characteristics, which could not be ruled out. Social desirability bias could not be ruled out. Despite these limitations, our analyses showed similar outcomes, and matched results in both arms favoured a digital HIVST over ConvHT.

Inherent risks of self-testing without an app-based guided program are namely: (A) failure of self-test as described in the self-test brochures, (B) failure to record and $\log$ their self-test result accurately, (C) failure to call for counselling when in need and (D) failure to seek linkage to ART care and initiation in nearest clinics. These risks exist for an unsupported program and cannot be discounted. Although app-based programs and web programs if adequately supported and maintained can minimise these risks, but they cannot necessarily eliminate them.

\section{Generalisability}

Our findings may be generalisable to young populations from South Africa and similar digitally savwy populations who can navigate care and counselling proactively, using an app-based program, guided by healthcare workers.

Our HIVST strategy is applicable in settings and in contexts where a committed team of counsellors is willing to work with the participants to customise their preferred choice of venue, language and linkage to clinics. Our study was restricted to Western Cape, which had a greater penetration of internet connectivity. Our recruiting clinics were served by the University of Cape Town, which has a formidable reputation in the city. The program was successful with a team of healthcare workers who were familiar with the patient populations they served and were able to navigate the healthcare systems with the study participants.

Some additional points of interest for implementers are highlighted below.

First, although we did not incentivise our program, we provided a flexible, choice-driven customised service that was greatly appreciated by participants. Flexibility and choice of venue and digital supports increased and maintained a continued engagement with our study participants. Second, this program will only work in settings where digital or Wi-Fi connectivity are uninterrupted, where participants are digitally savvy and can navigate an app-based service proactively. For those participants who had difficulty navigating their phones, we offered a choice of supervised strategy in clinics or kiosk based unsupervised HIVST strategy offered on tablets. Third, implementation challenges that were faced by us included the use of a protected HIPAA-compliant server that ran into firewall issues initially but took a month to resolve these issues. HIPAA-compliant servers are expensive but ensure data privacy and security. Fourth, some participants did not have the best Android phones (version five or higher), and they could not run the entire program on their phones due to bandwidth and storage issues, so they opted for the supervised HIVST in clinics or the kiosk based unsupervised HIVST. Fifth, mobilising ART initiation outside of UCT clinics required permission from the city. Sixth, the inherent sampling and social desirability bias in the study could impact generalisability of study findings. Those participants who desired to self-test using phones and apps consented to the study. Further exploration of these biases is needed to better customise these programs for young populations. Seventh, the continuous presence of UCT clinicians for ART initiation and care 
and presence of UCT trained counsellors and nurses for counselling and support for the duration of the project facilitated care. Lastly, mobilising and addressing the scepticism of some of our counsellors who were hesitant initially to embrace technology was an early challenge. The counsellors feared losing their jobs. However, as the study progressed, they engaged better with technology and found its relevance and its reach in serving populations they cared for and with speed and efficiency. After a few months, these counsellors became the champions of our program. Thus, by creating champions, the study generated clinical and public health impact data of relevance and generated social impact.

\section{Implications}

In 2021, in the setting of COVID-19 infection, recurrent lockdowns and COVID-19 variant induced epidemics are making it difficult to offer routine screening for HIV in public settings. Now, more than ever, there is an increased demand for HIVST with digital supports and related demand for increased screening for sexually transmitted and blood-borne infections. HIVST with digital support tools can pave the way for digital self-testing/self-sampling options for related screening initiatives for hepatitis C, COVID-19, Human Papillomavirus (HPV) and Chlamydiae Trachomatis /Neisseria Gonorrheae (CT/GC).

While barriers like smartphone ownership, digital literacy, access to $\mathrm{Wi}-\mathrm{Fi}$ and connectivity (digital divide) impede expanded access, they also offer opportunities to think of out-of-the-box ways to improve access. These include setting up public/private partnerships that offer a limited time ownership of smartphones or free Wi-Fi connectivity for a clinical or health service of a limited duration. Furthermore, flexible self-testing programs with digital supports should consider an affiliation with a credible organisation that offers a clinical service that is well regarded by the communities. Digital supports could be offered through flexible venues: online (through platforms), or through apps (smartphones), for homes or office use, or through kiosks in outreach clinics, malls, public and private pharmacies, or by non-government organisation-based outreach clinics, mobile vans or vending machines. ${ }^{46}$

Digital programs could be made available for open access for low-income countries and available for scale up in fast-track cities, through government support and smart public private partnerships. ${ }^{22}{ }^{50}$ Funded by the Canadian Institutes of Health Research (CIHR), in the coming years, we plan to expand our digital HIVST strategy for self-testing in key populations across Canadian provinces. ${ }^{51}$

\section{CONCLUSION}

Our choice-based, customised, self-tester centred, flexible digital HIVST program, offered by a dedicated team of healthcare workers and counsellors, successfully linked almost all self-testers to treatment and care. It detected new infections, reported an increase in referrals to HIVST and maintained engagement of participants for the duration of the project.

HIVST with digital support tools like smart apps and web platforms offers an untapped potential to fast track our progress towards UNAIDS targets for HIV elimination in many countries. Our findings find relevance in the planning and scaling of many self-testing and similar digital health initiatives worldwide.

\section{Author affiliations}

${ }^{1}$ Department of Medicine, McGill University, Montreal, Québec, Canada

${ }^{2}$ Research Institute of McGill University, Montreal, Québec, Canada

${ }^{3}$ South African MRC Centre for the Study of Antimicrobial Resistance, University of Cape Town, Cape Town, South Africa

${ }^{4}$ Centre for Lung Infection and Immunity, Division of Pulmonology, Department of Medicine and UCT Lung Institute, University of Cape Town, Cape Town, South Africa ${ }^{5}$ Department of Epidemiology, Biostatistics \& Occupational Health, McGill University, Montreal, Québec, Canada

${ }^{6}$ Department of Experimental Medicine, McGill University, Montreal, Québec, Canada

${ }^{7}$ Department of Health, Ethics \& Society/CAPHRI, Faculty of Health Medicine and Life Sciences, Maastricht University, Maastricht, The Netherlands

${ }^{8}$ School of Epidemiology \& Public Health, University of Ottawa, Ottawa, Ontario, Canada

${ }^{9}$ Faculty of Infectious and Tropical Diseases, Department of Infection Biology, London School of Hygiene and Tropical Medicine, London, UK

Twitter Nitika Pai @nikkiannike

Acknowledgements We duly acknowledge and thank all our study participants and deeply appreciate the hard work of our study staff members (healthcare workers, peer counsellors and nurses) of the University of Cape Town and our staff members at the RI-MUHC for their dedication to the project. We acknowledge and thank our study participants for their participation and time.

Contributors NP, AE and KD conceptualised and designed the research study. NP, $A E, S O, M P, J D, M S, N K, A d W$ and $K D$ conducted the research and investigation. $\mathrm{NP}, \mathrm{AE}, \mathrm{MP}, \mathrm{JD}, \mathrm{NK}, \mathrm{AdW}$ and KD contributed essential study materials and analysis tools. NP, AE, SO, JD, MS and KD coordinated research activities. PSC, GM and MF curated and analysed the data. NPP, AE, PSC, NE, AZ and KD were involved in design of methodology. NP, AE, PSC and KD wrote and critically revised the first draft of the manuscript. All authors read and approved the final version.

Funding This work was supported by operating grants $0732-05$ and 0710-05 from Grand Challenges Canada (funded by Government of Canada). As part of comatching fund requirements, grant support was provided by: (1) the Government of South Africa, through its Medical Research Council SHIP programme and the Department of Science and Technology and (2) the McGill University Health Centre Foundation. Kit donation and support was provided by (3) OraSure Technologies Inc, Bethlehem; Dr Pant Pai also acknowledges the support of Fonds de recherche du Quebec - Santé Research Scholar Awards (Junior and Senior), and Canadian Institutes of Health Research grants (HHP 137872, PJT 153149) for the duration of the project. KD acknowledges funding from the SA MRC (RFA-EMU-02-2017), EDCTP (TMA-2015SF-1043, TMA-1051-TESAll, TMA-CDF2015), UK Medical Research Council (MR/S03563X/1) and the Wellcome Trust (MR/S027777/1).

Competing interests HIVSmart! is an open access application that is owned by Grand Challenges Canada, McGill University and Dr Pant Pai.

Patient and public involvement Patients and/or the public were not involved in the design, or conduct, or reporting, or dissemination plans of this research.

Patient consent for publication Not required.

Ethics approval We obtained institutional review board approvals from the Research Institute of the McGill University Health Centre, the University of Cape Town, and research permits from the City of Cape Town. All participants provided written informed consent.

Provenance and peer review Not commissioned; externally peer reviewed.

Data availability statement Data are available on reasonable request. All data relevant to the study are included in the article or uploaded as supplementary 
information. All data relevant to the study are included in the article or uploaded as supplementary information.

Open access This is an open access article distributed in accordance with the Creative Commons Attribution 4.0 Unported (CC BY 4.0) license, which permits others to copy, redistribute, remix, transform and build upon this work for any purpose, provided the original work is properly cited, a link to the licence is given, and indication of whether changes were made. See: https://creativecommons.org/ licenses/by/4.0/.

ORCID iD

Nitika Pai http://orcid.org/0000-0002-4672-0500

\section{REFERENCES}

1 World Health Organization. Guidelines on HIV self-testing and partner notification. supplement to consolidated guidelines on HIV testing services. Available: https://apps.who.int/iris/bitstream/ handle/10665/251655/9789241549868-eng.pdf;jsessionid=5B2B C28C51DB096B58B8133BA8D9CB97?sequence=1 [Accessed 9 Sep 2020].

2 Unitaid. Market and technology landscape: HIV rapid diagnostic tests for self-testing, 4 EDN. Available: https://unitaid.org/assets/ HIVST-landscape-report.pdf [Accessed 9 Sep 2020].

3 Pai NP, Dheda K. Hiv self-testing strategy: the middle road. Expert Rev Mol Diagn 2013;13:639-42.

4 Pant Pai N, Sharma J, Shivkumar S, et al. Supervised and unsupervised self-testing for HIV in high- and low-risk populations: a systematic review. PLoS Med 2013;10:e1001414.

5 Qin Y, Han L, Babbitt A, et al. Experiences using and organizing HIV self-testing. AIDS 2018;32:371-81.

6 Krause J, Subklew-Sehume F, Kenyon C, et al. Acceptability of HIV self-testing: a systematic literature review. BMC Public Health 2013;13:735.

7 Jakobsen SF, Raben D, Sperle I. Scale-Up of HIV self-testing. HIV Med 2018.

8 Jin X, Xu J, Smith MK, et al. An Internet-based Self-Testing model (easy test): cross-sectional survey targeting men who have sex with men who never tested for HIV in 14 provinces of China. J Med Internet Res 2019;21:e11854.

9 Wray TB, Chan PA, Simpanen E, et al. A pilot, randomized controlled trial of HIV Self-Testing and real-time Post-Test Counseling/Referral on screening and preventative care among men who have sex with men. AIDS Patient Care STDS 2018;32:360-7.

10 Katz DA, Golden MR, Hughes JP, et al. Hiv Self-Testing increases HIV testing frequency in high-risk men who have sex with men: a randomized controlled trial. J Acquir Immune Defic Syndr 2018;78:505-12

11 Cambiano V, Johnson CC, Hatzold K, et al. The impact and costeffectiveness of community-based HIV self-testing in sub-Saharan Africa: a health economic and modelling analysis. J Int AIDS Soc 2019;22 Suppl 1:e25243.

12 Mangenah C, Mwenge L, Sande L, et al. Economic cost analysis of door-to-door community-based distribution of HIV Self-Test kits in Malawi, Zambia and Zimbabwe. J Int AIDS Soc 2019;22 Suppl $1: \mathrm{e} 25255$.

13 Stevens DR, Vrana CJ, Dlin RE, et al. A global review of HIV Selftesting: themes and implications. AIDS Behav 2018;22:497-512.

14 World Health Organization. Innovative who HIV testing recommendations AIM to expand treatment coverage. Available: https://www.who.int/news-room/detail/27-11-2019-innovative-whohiv-testing-recommendations-aim-to-expand-treatment-coverage [Accessed 9 Sep 2020].

15 UNAIDS. Miles to go: closing gaps, breaking barriers, righting injustices. Available: https://www.unaids.org/en/resources/ documents/2018/global-aids-update [Accessed 9 Sep 2020].

16 National Department of Health. Cost and impact of HIV selfscreening (HIVSS) in South Africa (preliminary report. Pretoria: National Department of Health, 2018.

17 World Health Organization. Post-Market surveillance of in vitro diagnostics. Available: https://apps.who.int/iris/bitstream/handle/ 10665/255576/9789241509213-eng.pdf?sequence=1\&isAllowed=y [Accessed 9 Sep 2020]

18 Hoagland B, Torres TS, Bezerra DRB. Telemedicine as a tool for PreP delivery during the COVID-19 pandemic in a large HIV prevention service in Rio de Janeiro-Brazil. Braz J Infect Dis 2020.

19 Mhango M, Chitungo I, Dzinamarira T. COVID-19 Lockdowns: impact on Facility-Based HIV testing and the case for the scaling up of home-based testing services in sub-Saharan Africa. AIDS Behav 2020:1-3.

20 Chenneville T, Gabbidon K, Hanson P, et al. The impact of COVID-19 on HIV treatment and research: a call to action. Int J Environ Res Public Health 2020;17. doi:10.3390/ijerph17124548. [Epub ahead of print: 2406 2020].

21 Accelerating Science Award Program. Hiv self-test empowers patients. Available: http://asap.plos.org/finalists/hiv-self-testempowers-patients/ [Accessed 9 Sep 2020].

22 Pant Pai N, Behlim T, Abrahams L, et al. Will an unsupervised selftesting strategy for HIV work in health care workers of South Africa? A cross sectional pilot feasibility study. PLoS One 2013;8:e79772.

23 Pant Pai N, Bhargava M, Joseph L, et al. Will an unsupervised selftesting strategy be feasible to operationalize in Canada? results from a pilot study in students of a large Canadian university. AIDS Res Treat 2014;2014:1-8

24 Pant Pai N, Smallwood M, Desjardins L, et al. An unsupervised smart App-Optimized HIV Self-Testing program in Montreal, Canada: cross-sectional study. J Med Internet Res 2018;20:e10258.

25 Austin PC. The performance of different propensity-score methods for estimating relative risks. J Clin Epidemiol 2008;61:537-45.

26 Phanuphak N, Anand T, Jantarapakde J, et al. What would you choose: online or Offline or mixed services? feasibility of online HIV counselling and testing among Thai men who have sex with men and transgender women and factors associated with service uptake. $J$ Int AIDS Soc 2018;21:e25118.

27 Wang Z, Lau JTF, Ip M, et al. A randomized controlled trial evaluating efficacy of promoting a home-based HIV Self-Testing with online counseling on increasing HIV testing among men who have sex with men. AIDS Behav 2018;22:190-201.

28 Zhong F, Tang W, Cheng W, et al. Acceptability and feasibility of a social entrepreneurship testing model to promote HIV self-testing and linkage to care among men who have sex with men. HIV Med 2017;18:376-82.

29 Green KE, Vu BN, Phan HT, et al. From conventional to disruptive: upturning the HIV testing status quo among men who have sex with men in Vietnam. J Int AIDS Soc 2018;21 Suppl 5:e25127.

$30 \mathrm{Ng}$ OT, Chow AL, Lee VJ, et al. Accuracy and user-acceptability of HIV self-testing using an oral fluid-based HIV rapid test. PLoS One 2012;7:e45168

31 De Boni RB, Lentini N, Santelli AC, et al. Self-testing, communication and information technology to promote HIV diagnosis among young gay and other men who have sex with men (MSM) in Brazil. J Int AIDS Soc 2018;21 Suppl 5:e25116.

32 LeGrand S, Muessig KE, Horvath KJ, et al. Using technology to support HIV self-testing among MSM. Curr Opin HIV AIDS 2017;12:425-31.

33 MacGowan RJ, Chavez PR, Gravens L, et al. Pilot evaluation of the ability of men who have sex with men to self-administer rapid HIV tests, prepare dried blood spot cards, and interpret test results, Atlanta, Georgia, 2013. AIDS Behav 2018;22:117-26.

34 Rosengren AL, Huang E, Daniels J, et al. Feasibility of using Grindr ${ }^{\mathrm{TM}}$ to distribute HIV self-test kits to men who have sex with men in Los Angeles, California. Sex Health 2016. doi:10.1071/SH15236. [Epub ahead of print: 23 May 2016].

35 Bell SFE, Dean JA, Lemoire J, et al. Integrated HIV self-testing (HIVST) service delivery in Queensland for policy and service development: study protocol. AIDS Care 2019;31:207-15.

36 Koutentakis K, Hoyos J, Rosales-Statkus M-E, et al. HIV self-testing in Spain: a valuable testing option for men-who-have-sex-with-men who have never tested for HIV. PLoS One 2019;14:e0210637.

37 Pettifor A, MacPhail C, Hughes JP, et al. The effect of a conditional cash transfer on HIV incidence in young women in rural South Africa (HPTN 068): a phase 3, randomised controlled trial. Lancet Glob Health 2016;4:e978-88.

38 Janssen R, Engel N, Esmail A, et al. Alone but supported: a qualitative study of an HIV Self-testing APP in an observational cohort study in South Africa. AIDS Behav 2020;24:467-74.

39 Janssen R, Engel N, Pant Pai N, et al. 'You're only there on the phone'? A qualitative exploration of community, affect and agential capacity in HIV self-testing using a smartphone APP. Sociol Health IIIn 2021;43:591-606.

40 Pant Pai N, Daher J, Prashanth HR, et al. Will an innovative connected AideSmart! app-based multiplex, point-of-care screening strategy for HIV and related coinfections affect timely quality antenatal screening of rural Indian women? results from a crosssectional study in India. Sex Transm Infect 2019;95:133-9.

41 Harrison A, Colvin CJ, Kuo C, et al. Sustained high HIV incidence in young women in southern Africa: social, behavioral, and structural factors and emerging intervention approaches. Curr HIVIAIDS Rep 2015;12:207-15. 
42 Masters SH, Agot K, Obonyo B, et al. Promoting partner testing and couples testing through secondary distribution of HIV Self-Tests: a randomized clinical trial. PLoS Med 2016;13:e1002166.

43 Thirumurthy $\mathrm{H}$, Masters SH, Mavedzenge SN, et al. Promoting male partner HIV testing and safer sexual decision making through secondary distribution of self-tests by HIV-negative female sex workers and women receiving antenatal and post-partum care in Kenya: a cohort study. Lancet HIV 2016;3:e266-74.

44 Korte JE, Kisa R, Vrana-Diaz CJ, et al. Hiv oral Self-Testing for male partners of women attending antenatal care in central Uganda: uptake of testing and linkage to care in a randomized trial. $J$ Acquir Immune Defic Syndr 2020;84:271-9.

45 Gichangi A, Wambua J, Mutwiwa S, et al. Impact of HIV SelfTest distribution to male partners of ANC clients: results of a randomized controlled trial in Kenya. J Acquir Immune Defic Syndr 2018;79:467-73.

46 World Health Organization. Hiv self-testing strategic framework: a guide for planning, introducing and scaling up. Available: https:// apps.who.int/iris/bitstream/handle/10665/275521/9789241514859eng.pdf?ua=1 [Accessed 9 Sept 2020].

47 MacGowan RJ, Chavez PR, Borkowf CB, et al. Effect of InternetDistributed HIV Self-tests on HIV diagnosis and behavioral outcomes in men who have sex with men. JAMA Intern Med 2020;180:117-25.

48 Steinhubl SR, Wolff-Hughes DL, Nilsen W, et al. Digital clinical trials: creating a vision for the future. NPJ Digit Med 2019;2:126.

49 CAHR. An epidemiological model for HIV Self-testing in Canada. 29th Annual Canadian Conference on HIVIAIDS Research (CAHR), 2020.

50 Med e-News. IAPAC, RI-MUHC, SYMPACT-X announce partnership to implement HIVSmart! TM self-testing app in high HIV burden fasttrack cities. Available: https://publications.mcgill.ca/medenews/ 2017/09/18/apac-ri-muhc-sympact-x-announce-partnership-toimplement-hivsmart-self-testing-app-in-high-hiv-burden-fast-trackcities/ [Accessed 9 Sep 2020].

51 Rourke SB, Pant Pai N, Kim J. Testing, reaching the undiagnosed and linkages to care: "REACHing" for impact. CIHR Team Grant: HIV/AIDS Biomedical and Clinical Research - Prevention, 2020. 